Gomez, A., Gonzales, S., \& Francia-Quiroz, J. C. (2021). Efecto de la temperatura y concentración de microplásticos en la tasa de filtración del mejillón Semimytilus algosus (Mytiloida: Mytilidae). Revista de Biología Tropical, 69(4), 1242-1251. https://doi.org/10.15517/rbt.v69i4.45426

\title{
Efecto de la temperatura y concentración de microplásticos en la tasa de filtración del mejillón Semimytilus algosus (Mytiloida: Mytilidae)
}

\author{
Angelo Gomez ${ }^{1 *}$, $;$ (D) https://orcid.org/0000-0003-1573-6160 \\ Sergio Gonzales ${ }^{1,2}$; (D) https://orcid.org/0000-0002-6357-033X \\ Juan C. Francia-Quiroz ; (iD https://orcid.org/0000-0001-5095-6623 \\ 1. Facultad de Ciencias Veterinarias y Biológicas, Universidad Científica del Sur, Av. Antigua Carretera Panamericana \\ Sur Km 19, Villa El Salvador, Lima 42, Perú; angelo1996biomar@gmail.com (*Correspondencia), \\ s_gonzalesb@outlook.com,jfrancia@cientifica.edu.pe \\ 2. Coastal Ecosystems of Peru Research Group, Universidad Científica del Sur, Lima, Perú.
}

Recibido 14-I-2021. Corregido 08-X-2021. Aceptado 17-XI-2021.

\author{
ABSTRACT \\ Effect of temperature and microplastic concentration on the filtration rate \\ of the mussel Semimytilus algosus (Myiloida: Mytilidae)
}

\begin{abstract}
Introduction: The presence of microplastics (MPs, particles smaller than $5 \mathrm{~mm}$ ) and the increase in temperature in the oceans, have been generating disturbances in marine life, which can be related to alterations in the metabolism of filter-feeders, such as Mythilids.

Objective: The effect of different temperatures and concentrations of MPs on the filtration rate (TF) of Semimytilus algosus is evaluated.

Methods: A sample of organisms $(\mathrm{N}=72)$ was exposed to four temperatures $\left(17,20,23\right.$ and $\left.26{ }^{\circ} \mathrm{C}\right)$, and a control without microplastics $\left(\mathrm{MPs}_{0}\right)$ and two concentrations of MPs $(<125 \mu \mathrm{m})$ of $0.125 \mathrm{mg} / 1\left(\mathrm{MPs}_{1}\right)$ and 0.250 $\mathrm{mg} / 1\left(\mathrm{MPs}_{2}\right)$, all in combination with Isochrysis galbana microalgae $\left(1 \times 10^{6} \mathrm{cell} / \mathrm{s} / \mathrm{ml} / \mathrm{day}\right)$ for 21 days.

Results: As the concentration of MPs increased, the TF of S. algosus decreased. Regarding temperature, during day 7 a higher TF was observed at $23{ }^{\circ} \mathrm{C}$ in all treatments, and during days 14 and 21 the lowest TF values were obtained at 23 and $26^{\circ} \mathrm{C}$. The joint action of the increase in temperature and MPs, negatively affected the TF of $S$. algosus, where both factors caused the decrease in TF for all evaluation times. No mortality was recorded at $17^{\circ} \mathrm{C}$ for any treatment, and in the case of mytylids exposed to $\mathrm{MPs}_{1}$ at $20{ }^{\circ} \mathrm{C}$ and $26{ }^{\circ} \mathrm{C}$, the highest mortality (67\%) occurred.
\end{abstract}

Conclusions: The study demonstrates the adverse effect of the increase in temperature and MPs on the TF of S. algosus.

Key words: marine mussel; metabolism; polyethylene terephthalate; plastic; global warming.

La elaboración de plástico a nivel global es de 300 millones de toneladas al año, donde aproximadamente el $50 \%$ es eliminado al medio ambiente (Manchala \& Ramana, 2020). La acumulación de estos residuos, cerca de los ríos, facilita el transporte del plástico a otros cuerpos de agua, lo que permite que se depositen en los océanos (Horton et al., 2017). La contaminación por plásticos es una problemática que afecta el estado de los hábitats marinos y su composición biológica (Avio et al., 2017). El fraccionamiento natural y artificial de los plásticos ha dado como resultado partículas sólidas llamadas microplásticos (MPs, $<5 \mathrm{~mm}$ ) 
(GESAMP, 2015), como producto de procesos físicos causados por la radiación ultravioleta (UV), abrasión e interacción con las corrientes oceánicas (MPs secundarios) (Frias \& Nash, 2019). Además, los MPs se originan por la fabricación de productos de cuidado personal (usados como exfoliantes), y la aplicación en procesos de granallado y relleno de algunos productos textiles (MPs primarios) (GESAMP, 2015). La presencia de MPs, se ha reportado en playas (De-la-Torre et al., 2020), sedimentos (Courtene-Jones et al., 2020), aguas superficiales (Li et al., 2020) y organismos marinos (Walkinshaw et al., 2020); es a partir de aquí que los estudios han evidenciado que los MPs generan efectos perjudiciales sobre procesos fisiológicos como alimentación, reproducción y crecimiento (Botterell et al., 2019).

Los moluscos bivalvos adquieren alimento y energía de su entorno a través de la filtroalimentación, con la captura de partículas de reducido tamaño (microseston), lo cual los hace buenos indicadores del efecto de los MPs sobre ecosistemas acuáticos (Rosa et al., 2018). Es así, como diversos trabajos, han demostrado las consecuencias negativas de los MPs en moluscos, donde se ha reportado malformaciones en el crecimiento para Mytilus edulis (Rist et al., 2019), alteraciones en la demanda de alimento y eficiencia de absorción de Crassostrea gigas (Sussarellu et al. 2016), e incremento en la tasa de respiración en Ostrea edulis (Green, 2016).

El cambio climático se ha convertido en uno de los tópicos más relevantes de los últimos años, debido a la influencia que ejerce en el incremento de la temperatura de los océanos según la Organización Meteorológica Mundial (World Meteorological Organization, 2019). Esto ha generado la alteración de la sensibilidad en la tolerancia térmica de los organismos marinos, especialmente de especies con nichos ecológicos conservados a nivel local (Bennett et al., 2019), como es el caso de los invertebrados (González et al., 2014). Se pueden apreciar los efectos del incremento de la temperatura, por ejemplo, en Musculista senhousia y Limnoperna fortunei, con perturbaciones de sus tasas de filtración (TF) (Inoue \& Yamamuro,
2000; Sylvester et al., 2005); mientras que, en Mytilus edulis, se ha reportado el incremento de la mortalidad post-desove por temperaturas elevadas (Clements et al., 2018).

El efecto combinado de temperatura y MPs ha sido estudiado en invertebrados acuáticos, como: Daphnia magna, donde se vio afectada negativamente su supervivencia y capacidad de filtración (Serra et al., 2020), y Gammarus pulex que presentó alteraciones de la tasa de respiración (Kratina et al., 2019). Dentro de este escenario, los organismos sésiles como los bivalvos podrían ser afectados por el efecto conjunto del incremento de temperatura y la contaminación por MPs, ocasionando alteraciones metabólicas como las reportadas en Mytilus trossulus y Crassostrea gigas (Lenz, 2016).

Dentro de la lista de bivalvos del Perú se encuentran Semimytilus algosus (Paredes et al., 2016) formando parte de los ecosistemas costeros rocosos (Paredes et al., 1999). Este organismo se encuentra posicionado y agrupado en el intermareal, respecto a sus adaptaciones fisiológicas (Brante et al., 2019), formando frondas calcáreas que son el refugio de otras especies (Paredes et al., 1999). Además, es parte de la trasferencia energética, los cuales constituyen parte de la dieta de especies de interés comercial como Mugil cephalus, Sciaena deliciosa y Paralonchurus peruanus (Fernández, 2014; Gutiérrez-Díaz, 2017; Pérez-Carrasco, 2017). La recolecta local de este organismo se debe a su musculatura, y es utilizada como carnada para la pesca artesanal (Molinet et al., 2007). Por lo tanto, la función ecológica y comercial están sustentadas por densidades predominantes que logran formarse en espacios representativos en el litoral marino, los cuales están expuestos a cualquier tipo de contaminación costero como la de los MPs y exposiciones térmicas extremas (Alfaro-Mudarra et al., 2016; Tejada-Pérez et al., 2018). En este contexto, el presente estudio tuvo como objetivo evaluar el efecto de diferentes temperaturas y concentraciones de MPs sobre la TF de Semimytilus algosus. 


\section{MATERIALES Y MÉTODOS}

Obtención y aclimatación de organismos: Los ejemplares de $S$. algosus (72 individuos, $40.5 \pm 1.15 \mathrm{~mm}$ ) fueron recolectados de la orilla rocosa (submareal) de la playa San Bartolo (balneario) el 15 de julio del 2019 en la estación de invierno (Lima, Perú) (12²3'22.48" $\mathrm{S} \& 76^{\circ} 46^{\prime} 57.44^{\prime}$ W). Los organismos fueron transportados en envases plásticos de 201 conteniendo agua de mar al Laboratorio de Larvicultura Experimental de la Universidad Científica del Sur, donde se acondicionaron por 7 días (Filgueira et al., 2009) con aireación constante y condiciones de agua de mar de 18 $\pm 1{ }^{\circ} \mathrm{C}$ y $35 \mathrm{ppm}$ de salinidad (Pinochet et al., 2018). Los organismos fueron alimentados con la microalga Isochrysis galbana $\left(1 \times 10^{6} \mathrm{cel} / \mathrm{ml} /\right.$ día), siguiendo el protocolo de Pinochet et al. (2018) durante la aclimatación y experimentación. Previo al experimento, los individuos de S. algosus se aclimataron a las cuatro temperaturas $\left(17,20,23\right.$ y $\left.26^{\circ} \mathrm{C}\right)$, considerando las estimaciones térmicas del Grupo Intergubernamental de Expertos sobre el Cambio Climático (IPCC, 2014; IPCC, 2018) e Instituto del Mar del Perú (2017). Se tomó un día para el acondicionamiento a $17{ }^{\circ} \mathrm{C}$, mientras que para las otras temperaturas se aumentó $1{ }^{\circ} \mathrm{C}$ por día, durante 8 días (Filgueira et al., 2009).

Preparación de los microplásticos (MPs): Los MPs se obtuvieron frotando una lija metálica (\# 150) sobre botellas nuevas de tereftalato de polietileno (PET) (esto se indicaba en la base de la botella) (Erni-Cassola et al., 2017). El trabajo se desarrolló en un ambiente separado, dentro de una caja cubierta con un envoltorio plástico que solo permitía el ingreso de las manos para lijar las botellas. En la base, se ubicó una placa Petri de 150 $\mathrm{mm}$, con papel aluminio y sobre este un tamiz metálico de $125 \mu \mathrm{m}$ para obtener partículas menores a esta medida (Munno et al., 2017). Únicamente se trabajó con la placa Petri cerrada, la cual fue llevada con el material tamizado a una balanza analítica, donde se separó con un pincel y se pesó cada concentración de MPs en placas Petri de $100 \mathrm{~mm}$ con papel aluminio respectivamente. Este procedimiento se repitió hasta conseguir las cantidades necesarias para el recambio de partículas. Previamente a cada recambio se separaron envases con agua para cada temperatura para evitar el estrés térmico en los organismos. Además, con el fin de evitar la contaminación de las partículas a otras unidades experimentales, durante el proceso de obtención de las partículas y exposición de estas mismas, las unidades experimentales sin MPs se mantuvieron cerradas, únicamente se hizo un agujero para el ingreso del conducto de aireación. Por otro lado, para asegurar la exposición y suspensión de los MPs se mantuvo la manguera de aireación hasta el fondo de los vasos de vidrio (500 $\mathrm{ml}$ de capacidad); con ello, las partículas se mantuvieron en constante resuspensión en la columna de agua. Esto de definió a partir de la densidad del MPs PET de $1.38 \mathrm{mg} / \mathrm{l}$, la cual es mayor a la densidad del agua de mar $(1.025 \mathrm{mg} / \mathrm{l})$

Diseño experimental: Los ejemplares fueron colocados en unidades experimentales de vidrio de 0.51 , y se expusieron por 21 días a dos concentraciones de MPs en combinación con la microalga $I$. galbana $\left(1 \times 10^{6} \mathrm{cel} / \mathrm{ml} /\right.$ día $)$. Los tratamientos fueron: Un control, $\mathrm{MPs}_{0}=$ I. galbana sin MPs, concentración $1, \mathrm{MPs}_{1}=$ I. galbana y $0.125 \mathrm{mg} / \mathrm{l}$ de MPs, y concentración $2, \mathrm{MPs}_{2}=I$. galbana y $0.250 \mathrm{mg} / \mathrm{l} \mathrm{de}$ MPs, para cada una de las cuatro temperaturas experimentales $\left(17,20,23\right.$ y $26^{\circ} \mathrm{C}$ ) (Fig. 1). Las concentraciones de MPs fueron tomadas a partir de la densidad de partículas plásticas descrita para las islas de basura en el Océano Pacífico (Goldstein et al., 2013; Elías, 2015). Además, se realizó la renovación de agua y partículas de MPs tres veces por semana, con la finalidad de mantener limpia el agua de mar y concentraciones de MPs (Bråte et al., 2018).

Tasa de Filtración (TF): Para determinar variaciones de la capacidad de filtración de $S$. algosus por efecto del incremento de temperatura y MPs, se evaluó la TF cada 7 días durante 1 hora (Bøhle, 1972). Primero, los mitílidos se 


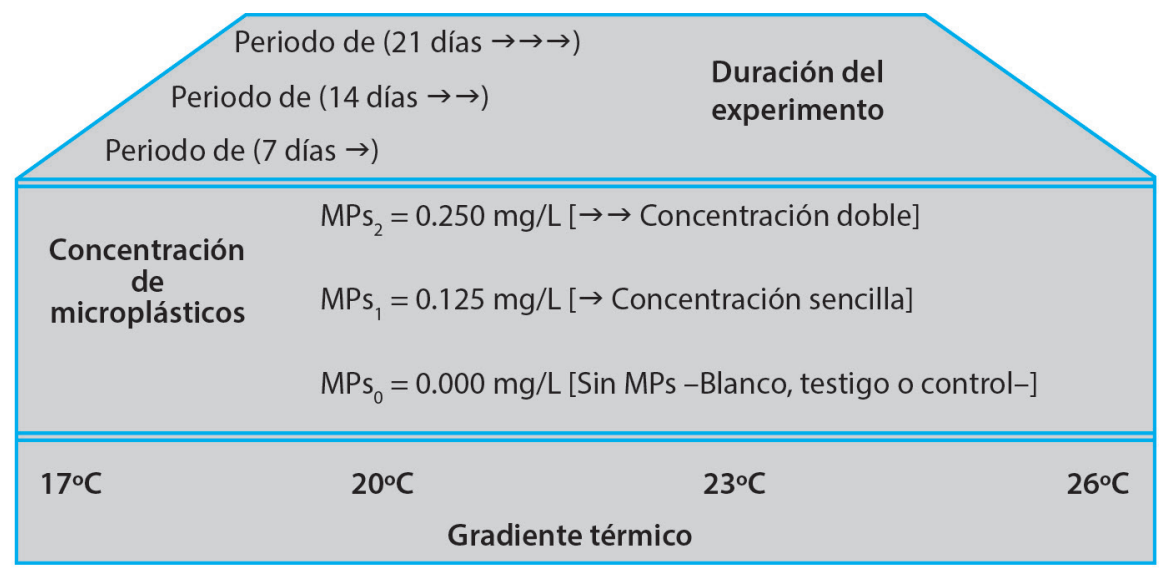

Fig. 1. Diseño experimental. Se indica a la temperatura en grados centígrados ${ }^{\circ} \mathrm{C}$, tres concentraciones de microplásticos (MPs) y los días de toma de muestras como periodos de evaluación.

Fig. 1. Experimental design. The temperature in degrees centigrade ${ }^{\circ} \mathrm{C}$, three concentrations of microplastics (MPs) and the days of sampling are indicated as evaluation periods.

trasladaron a unidades experimentales de 0.51 con aireación constante, según las temperaturas por tratamiento, y se mantuvieron sin alimento por 24 horas previo a la evaluación de TF. Luego, se estimó la TF utilizando la microalga I. galbana $\left(1 \times 10^{6} \mathrm{cel} / \mathrm{ml}\right)$. Para evaluar la TF se aplicó la fórmula de Jørgensen (1996):

$$
\mathrm{TF}=\mathrm{V} *(\log \mathrm{Ci}-\log \mathrm{C}) /(0.434 * \mathrm{~T})
$$

Donde: TF es la Tasa de Filtración (mL/ind/h), $\mathrm{Ci}$ es la concentración de microalga inicial, $\mathrm{Cf}$ es la concentración de microalga final, 0.434 es el valor de logaritmo de e y $\mathrm{T}$ es el tiempo en horas $(\mathrm{h})$.

Mortalidad (\%): Luego de 21 días de exposición a las diferentes temperaturas y concentraciones de MPs, se determinó la mortalidad dividiendo el número de organismos muertos al final del experimento entre el número inicial de organismos (Freitas et al., 2019).

Análisis estadístico: Los análisis estadísticos se realizaron utilizando el programa estadístico IBM SPSS versión 25.0 para Windows. En el análisis de prerrequisitos, se comprobó la normalidad (test de Shapiro Wilk) y homocedasticidad (test de Levene) para los datos de la TF (mL/ind/h). A partir de satisfacer estas condiciones, se empleó la prueba de ANOVA III (de tres vías) para demostrar diferencias significativas $(\alpha \leq 0.05)$ del efecto separado y conjunto de ambos factores (temperatura y MPs) y el tiempo, además de la prueba de Tukey para las comparaciones múltiples por tratamiento para la $\mathrm{TF}(\mathrm{ml} / \mathrm{ind} / \mathrm{h})$ en el tiempo (días).

\section{RESULTADOS}

A los 21 días de experimentación, se determinó que la presencia de MPs ocasionó variaciones en la TF de $S$. algosus $(\mathrm{F}=244.36$, g.1. = $2, \mathrm{P}<0.05)$. Esto se evidenció para individuos del tratamiento $\mathrm{MPs}_{1}($ Media $=10.76 \pm 3.86$ $\mathrm{ml} / \mathrm{ind} / \mathrm{h}){\text { y } \mathrm{MPs}_{2}}_{2}($ Media $=7.67 \pm 3.19 \mathrm{ml} /$ $\mathrm{ind} / \mathrm{h}$ ), donde ambas TF fueron inferiores al testigo $\sin$ MPs $($ Media $=14.81 \pm 4.57 \mathrm{ml} / \mathrm{ind} / \mathrm{h}$ ) $(\mathrm{P}<0.05)$. Durante el día 7 de evaluación, la menor TF fue para $\mathrm{MPs}_{2}($ Media $=10.75 \pm$ $2.29 \mathrm{ml} / \mathrm{ind} / \mathrm{h})$ frente al testigo, $\mathrm{MPs}_{0}($ Media $=$ $18.31 \pm 2.98 \mathrm{ml} / \mathrm{ind} / \mathrm{h})(\mathrm{P}<0.05)$. Esta reducción de la TF para $\mathrm{MPs}_{2}$ continuó para el día 14 (Media $=5.62 \pm 2.18 \mathrm{ml} / \mathrm{ind} / \mathrm{h}$ ) y día 21 (Media $=6.38 \pm 2.18 \mathrm{ml} / \mathrm{ind} / \mathrm{h}$ ) (Fig. 2). 


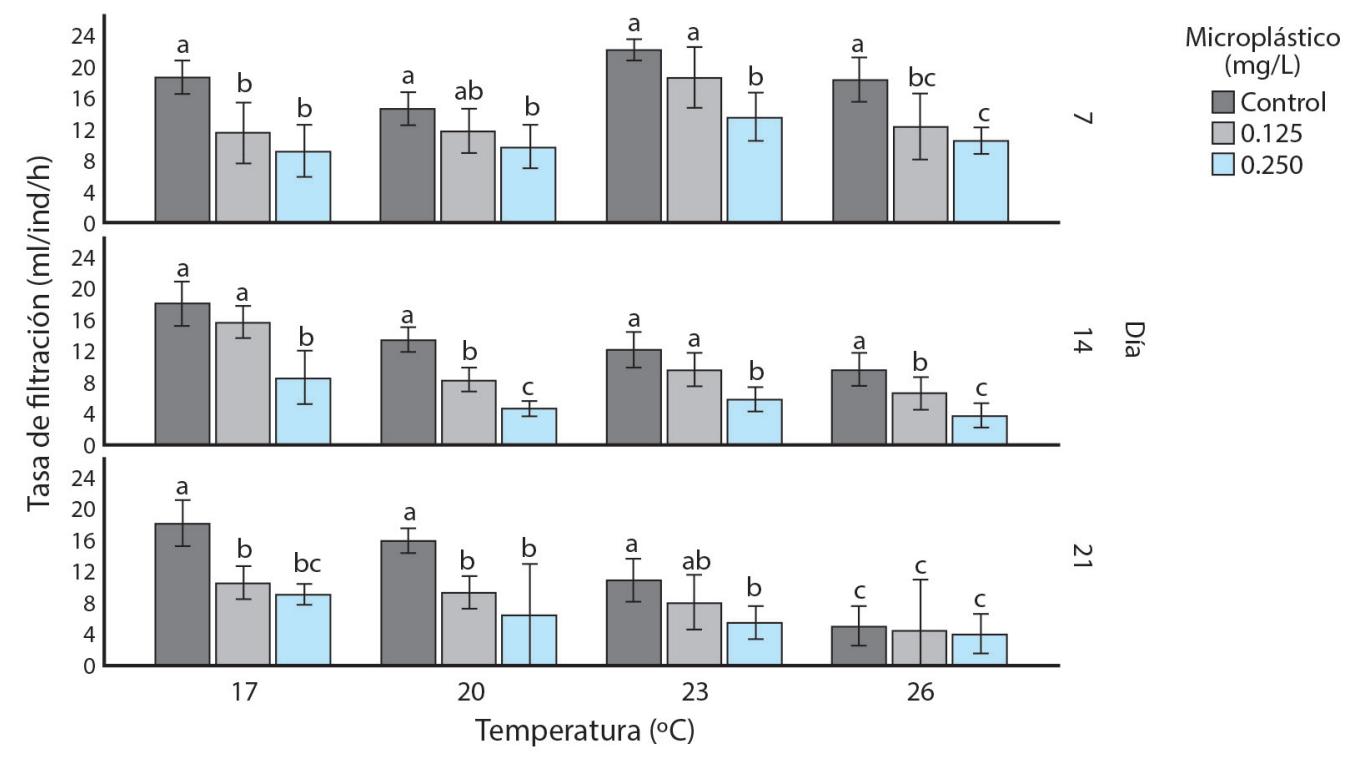

Fig. 2. Valores promedio de la Tasa de Filtración ( \pm desviación estándar, $\mathrm{N}=6$ ) para $S$. algosus, bajo el efecto de cuatro temperaturas $\left(17{ }^{\circ} \mathrm{C}, 20{ }^{\circ} \mathrm{C}, 23{ }^{\circ} \mathrm{C}\right.$ y $26^{\circ} \mathrm{C}$ ), evaluado contra el Testigo ( $\left.\mathrm{MPs}_{0}=0.00 \mathrm{mg} / \mathrm{l}\right)$ y dos concentraciones de MPs $\left(\mathrm{MPs}_{1}=0.125 \mathrm{mg} / 1\right.$ y $\left.\mathrm{MPs}_{2}=0.250 \mathrm{mg} / \mathrm{l}\right)$ y después de 7, 14 y 21 días de experimentación. Letras distintas indican diferencias significativas $(\mathrm{P}<0.05)$.

Fig. 2. Average values of the Filtration Rate ( \pm standard deviation, $\mathrm{N}=6$ ) for $S$. algosus, under the effect of four temperatures $\left(17^{\circ} \mathrm{C}, 20^{\circ} \mathrm{C}, 23^{\circ} \mathrm{C}\right.$ and $\left.26^{\circ} \mathrm{C}\right)$, evaluated against the Control (MP's $\left.\mathrm{s}_{0}=0.00 \mathrm{mg} / \mathrm{l}\right)$ and two concentrations of MPs (MPs $=0.125 \mathrm{mg} / 1$ and $\left.\mathrm{MPs}_{2}=0.250 \mathrm{mg} / \mathrm{l}\right)$ and after 7, 14 and 21 days of experimentation. Different letters indicate significant differences $(\mathrm{P}<0.05)$.

El incremento de temperatura redujo la $\mathrm{TF}$ de los bivalvos (ANOVA, $\mathrm{P}<0.05$ ), asimismo, se observaron diferencias significativas para todas las temperaturas $(\mathrm{F}=61.69$, g.l. $=3$, $\mathrm{P}<0.05)$. La TF de los organismos presentó variaciones a lo largo del estudio, donde los primeros 7 días de evaluación la TF fue menor a $20{ }^{\circ} \mathrm{C}$ (Media $=12 \pm 2.48 \mathrm{ml} / \mathrm{ind} / \mathrm{h}$ ) que a 23 ${ }^{\circ} \mathrm{C}($ Media $=18.00 \pm 4.00 \mathrm{ml} / \mathrm{ind} / \mathrm{h})$. Durante las evaluaciones de los días 14 y 21, se observó una reducción continua de la TF de 20 a 23 ${ }^{\circ} \mathrm{C}$, donde los menores valores fueron para los individuos sometidos a $26^{\circ} \mathrm{C}$ (Fig. 2)

El efecto combinado de temperatura y MPs afectó la TF de $S$. algosus $(\mathrm{F}=37.45$, g.l. $=6, \mathrm{P}$ $<0.05)$. Se evidenció que a mayor temperatura (de 20 a $26^{\circ} \mathrm{C}$ ) y aumento de la concentración de MPs se reduce la TF (Fig. 2). Al finalizar el experimento, no se observó mortalidad para ningún tratamiento a $17{ }^{\circ} \mathrm{C}$, mientras que la menor mortalidad en los individuos mantenidos $\sin$ MPs a $23{ }^{\circ} \mathrm{C}$, y la mayor cantidad de muertos sucedió en los tratamientos sin y con MPs a $26{ }^{\circ} \mathrm{C}, \mathrm{y} \mathrm{MPs}{ }_{2}$ a $20{ }^{\circ} \mathrm{C}$. (Tabla 1 ).

\section{DISCUSIÓN}

El presente estudio demostró que la presencia de MPs de PET $(<125 \mu \mathrm{m})$, a diferentes concentraciones $(0.125$ y $0.250 \mathrm{mg} / \mathrm{l})$, redujo la TF de $S$. algosus. Esto también se evidenció en otros bivalvos, donde el incremento en la concentración de MPs (fibras de PET de 500 $\mu \mathrm{m}$, de 3 a $30 \mathrm{MP}$ 's/l) ocasionó la disminución de la TF en Dreissena bugensis (Pedersen et al., 2020); y la exposición de fibras de PET (500 $\mu \mathrm{m}, 30 \mathrm{MPs} / \mathrm{ml})$ en $M$. edulis, afectó negativamente la TF (Woods et al., 2018). Estas alteraciones de la TF a causa de MPs, podrían explicarse por procesos de deciliación 
TABLA 1

Mortalidad (\%) de S. algosus a cuatro temperaturas $\left(17,20,23\right.$ y $\left.26{ }^{\circ} \mathrm{C}\right)$, en el Blanco o Testigo $\left(\mathrm{MPs}_{0}=0.00 \mathrm{mg} / \mathrm{L}\right)$ y dos concentraciones de MPs $\left(\mathrm{MPs}_{1}=0.125 \mathrm{mg} / 1\right.$ y $\left.\mathrm{MPs}_{2}=0.250 \mathrm{mg} / \mathrm{l}\right)$ expuesto por un periodo de 21 días $(\mathrm{N}=6), \mathrm{t}=$ tiempo

TABLE 1

Mortality (\%) of S. algosus at four temperatures (17, 20, 23 and $\left.26^{\circ} \mathrm{C}\right)$, on white or witness $\left(\mathrm{MPS}_{0}=0.00 \mathrm{mg} / \mathrm{l}\right)$ and two MPS concentrations $\left(\mathrm{MPS}_{1}=0.125 \mathrm{mg} / \mathrm{l}\right.$ and $\left.\mathrm{MPS}_{2}=0.250 \mathrm{mg} / \mathrm{l}\right)$ Exposed for a period of 21 days $(\mathrm{N}=6), \mathrm{t}=$ time

\begin{tabular}{ccc}
\hline Microplásticos (MPs) & Temperatura $\left({ }^{\circ} \mathrm{C}\right)$ & Mortalidad, $\mathrm{t}=21$ días $(\%)$ \\
\hline & 17 & 0 \\
MPs $_{0}$ & 20 & 0 \\
& 23 & 50 \\
& 26 & 0 \\
& 17 & 33 \\
MPs $_{1}$ & 20 & 33 \\
& 23 & 67 \\
& 26 & 0 \\
& 17 & 67 \\
& 20 & 0 \\
& 23 & 50 \\
\hline
\end{tabular}

e hipertrofia en branquias y glándula digestiva, respectivamente, causadas por el aumento en la concentración de MPs (Alnajar et al., 2020). Además, se puede considerar cierta retención de partículas de MPs durante la filtración e ingesta de alimento para S. algosus, dado que se ha reportado esta situación en bivalvos como Ennucula tenuis y Abra nitida, las cuales retuvieron partículas de MPs de tamaños similares a las utilizadas en nuestro estudio $(<125 \mu \mathrm{m})$, posterior a 4 semanas de exposición (Bour et al., 2018). Asimismo, se debe tener presente que los MPs se mantienen un mayor tiempo dentro del organismo al mezclarse con el alimento, como microalgas (Chae \& An, 2020).

En cuanto al efecto de la temperatura, durante la primera evaluación (7 días), la TF se incrementó a $23{ }^{\circ} \mathrm{C}$, pero a 20 y $26^{\circ} \mathrm{C}$ hubo una reducción de la TF. Esto se puede relacionar con un proceso de aclimatación que desarrollaron los organismos frente a la variación de temperatura (Widdows, 1978). Para los días 14 y 21, los individuos tuvieron una reducción de la TF a 23 y $26{ }^{\circ} \mathrm{C}$, lo cual pudo generar la reducción de la frecuencia de la apertura valvar, conducta natural de sobrevivencia de los mitílidos frente a condiciones adversas como el incremento de la temperatura (Weber et al., 2020).

Kratina et al. (2019), consideran que la interacción de MPs y temperatura se presenta de manera antagonista; sin embargo, nuestro estudio demostró que ambos factores $(0.125$ $\mathrm{mg} / \mathrm{l}$ y $0.250 \mathrm{mg} / \mathrm{l})\left(17,20,23\right.$ y $\left.26^{\circ} \mathrm{C}\right)$, dentro de los rangos límites para $S$. algosus, ocasionaron la reducción de la TF. Es así como, durante los días 14 y 21, se observó una reducción de la TF por efecto de ambos factores; es posible que el incremento de la $\mathrm{TF}$, provocada por el aumento de temperatura, haya inducido la ingesta de MPs en los primeros días de evaluación, lo cual magnificó el efecto negativo sobre la TF en los días posteriores.

Por otro lado, no se evidenció mortalidad para $S$. algosus en ausencia y presencia de MPs a $17^{\circ} \mathrm{C}$, así como para los individuos mantenidos como Blancos o Testigos a $\mathrm{MPs}_{0}$ para 20 ${ }^{\circ} \mathrm{C}$. Sin embargo, para estos organismos Blanco o Testigos MPs a 23 y $26^{\circ} \mathrm{C}$, se presentó una mortalidad del 17 y $50 \%$, respectivamente. Esto se podría relacionar con el rango de tolerancia térmica, donde Lenz et al. (2018) reportaron mortalidades del $70 \%$ a $28{ }^{\circ} \mathrm{C}$ para S. algosus. Además, las alteraciones originadas 
por el aumento de temperatura y la presencia de MPs, pueden ocasionar una menor ingesta de alimento, lo que lleva a los organismos a un estado de inanición (Rist et al., 2016), que se relaciona con las altas mortalidades reportadas para $\mathrm{MPs}_{1}$ a $26{ }^{\circ} \mathrm{C}$ y $\mathrm{MPs}_{2}$ para $20^{\circ} \mathrm{C}$.

Finalmente, el estudio demuestra que el incremento de la temperatura y la exposición a MPs, ocasiona una reducción de la TF, y genera mortalidad para $S$. algosus, que puede variar según el tiempo de exposición o evaluación. Esta situación puede ocasionar modificaciones en los ecosistemas marino-costeros, debido a que los bivalvos controlan el crecimiento de las poblaciones planctónicas y las concentraciones de micropartículas, a través del mecanismo de filtración (Marroni, 2019), y pueden transmitir los MPs a otros niveles de la cadena trófica, por la interrelación con sus depredadores (Farrell \& Nelson 2013; Li et al., 2019).

Declaración de ética: los autores declaran que todos están de acuerdo con esta publicación y que han hecho aportes que justifican su autoría; que no hay conflicto de interés de ningún tipo; y que han cumplido con todos los requisitos y procedimientos éticos y legales pertinentes. Todas las fuentes de financiamiento se detallan plena y claramente en la sección de agradecimientos. El respectivo documento legal firmado se encuentra en los archivos de la revista.

\section{AGRADECIMIENTOS}

Agradecemos al asistente técnico de laboratorio, Fernando Caldas Melchor, por su apoyo en el proceso experimental de la investigación.

\section{RESUMEN}

Introducción: La presencia de microplásticos (MPs, partículas menores a $5 \mathrm{~mm}$ ) y el incremento de la temperatura en los océanos, vienen generando perturbaciones en la vida marina, que se pueden relacionar con alteraciones en el metabolismo de organismos filtradores, como los mitílidos. Objetivo: Se evalúa el efecto de diferentes temperaturas y concentraciones de MPs sobre la tasa de filtración (TF) de Semimytilus algosus.
Métodos: Una muestra de organismos $(\mathrm{N}=72)$ fue expuesta a cuatro temperaturas $\left(17,20,23\right.$ y $\left.26{ }^{\circ} \mathrm{C}\right)$, y un testigo sin microplásticos $\left(\mathrm{MPs}_{0}\right)$ y dos concentraciones de MPs $(<125 \mu \mathrm{m})$ de $0.125 \mathrm{mg} / \mathrm{l}\left(\mathrm{MPs}_{1}\right)$ y $0.250 \mathrm{mg} / \mathrm{l}\left(\mathrm{MPs}_{2}\right)$, todos en combinación con la microalga Isochrysis galbana ( $1 \times 10^{6} \mathrm{cel} / \mathrm{ml} /$ día) por 21 días.

Resultados: A medida que aumentó la concentración de MPs, se redujo la TF de $S$. algosus. Respecto a la temperatura, durante el día 7 se observó una mayor TF a $23{ }^{\circ} \mathrm{C}$ en todos los tratamientos, y para los días 14 y 21 se obtuvieron los menores valores de TF a 23 y $26^{\circ} \mathrm{C}$. La acción conjunta del incremento de temperatura y MPs, afectó negativamente la TF de $S$. algosus, donde ambos factores ocasionaron el descenso de la TF para todos los tiempos de evaluación. No se registró mortalidad a $17{ }^{\circ} \mathrm{C}$ para ningún tratamiento, y en el caso de mitílidos expuestos a MPs $\mathrm{s}_{1}$ y temperaturas de 20 y $26{ }^{\circ} \mathrm{C}$ se presentó la mayor mortalidad (67\%).

Conclusiones: El estudio demuestra el efecto adverso del incremento de temperatura y MPs sobre la TF de $S$. algosus.

Palabras clave: mejillón marino; metabolismo; tereftalato de polietileno; plástico; calentamiento global.

\section{REFERENCIAS}

Alfaro-Mudarra, S., Rebaza-Castillo, V., De-Lucio-Burga, L., Salcedo-Rodríguez, J., \& Vásquez-Ruiz, C. (2016). Evaluación de bancos naturales de invertebrados marinos comerciales, Región La Libertad-Perú, 2012. Imarpe Perú, 43(1), 68-93.

Alnajar, N., Jha, A. N., \& Turner, A. (2020). Impacts of microplastic fibers on the marine mussel, Mytilus galloprovinciallis. Chemosphere, 262, 128290. https://doi.org/10.1016/j.chemosphere.2020.128290

Avio, C. G., Gorbi, S., \& Regoli, F. (2017). Plastics and microplastics in the oceans: From emerging pollutants to emerged threat. Marine Environmental Research, 128, 2-11. https://doi.org/10.1016/j. marenvres.2016.05.012

Bennett, S., Duarte, C. M., Marbà, N., \& Wernberg, T. (2019). Integrating within-species variation in thermal physiology into climate change ecology. Philosophical Transactions of the Royal Society B, 374(1778), 20180550. https://doi.org/10.1098/ rstb.2018.0550

Bøhle, B. (1972). Effects of adaptation to reduced salinity on filtration activity and growth of mussels (Mytilus edulis L.). Journal of Experimental Marine Biology and Ecology, 10(1), 41-47. https://doi. org/10.1016/0022-0981(72)90091-3

Botterell, Z. L., Beaumont, N., Dorrington, T., Steinke, M., Thompson, R. C., \& Lindeque, P. K. (2019). Bioavailability and effects of microplastics on marine 
zooplankton: A review. Environmental Pollution, 245, 98-110. https://doi.org/10.1016/j.envpol.2018.10.065

Bour, A., Haarr, A., Keiter, S., \& Hylland, K. (2018). Environmentally relevant microplastic exposure affects sediment-dwelling bivalves. Environmental Pollution, 236, 652-660. https://doi.org/10.1016/j. envpol.2018.02.006

Bråte, I. L. N., Blázquez, M., Brooks, S. J., \& Thomas, K. V. (2018). Weathering impacts the uptake of polyethylene microparticles from toothpaste in Mediterranean mussels (M. galloprovincialis). Science of the Total Environment, 626, 1310-1318. https://doi. org/10.1016/j.scitotenv.2018.01.141

Brante, A., Riera, R., \& Cartes, V. (2019). Post-settlement movement as response to interspecific competition between the bioengineer mussels Semimytilus algosus and Perumytilus purpuratus. Journal of Sea Research, 154, 101809. https://doi.org/10.1016/j. seares.2019.101809

Chae, Y., \& An, Y. J. (2020). Effects of food presence on microplastic ingestion and egestion in Mytilus galloprovincialis. Chemosphere, 240, 124855. https://doi. org/10.1016/j.chemosphere.2019.124855

Clements, J. C., Hicks, C., Tremblay, R., \& Comeau, L. A. (2018). Elevated seawater temperature, not pCO2, negatively affects post-spawning adult mussels (Mytilus edulis) under food limitation. Conservation Physiology, 6(1), 1-7. https://doi.org/10.1093/conphys/ $\operatorname{cox} 078$

Courtene-Jones, W., Quinn, B., Ewins, C., Gary, S. F., \& Narayanaswamy, B. E. (2020). Microplastic accumulation in deep-sea sediments from the Rockall Trough. Marine Pollution Bulletin, 154, 111092. https://doi.org/10.1016/j.marpolbul.2020.111092

De-la-Torre, G. E., Dioses-Salinas, D. C., Castro, J. M., Antay, R., Fernández, N. Y., Espinoza-Morriberón, D., \& Saldaña-Serrano, M. (2020). Abundance and distribution of microplastics on sandy beaches of Lima, Peru. Marine Pollution Bulletin, 151, 110877. https://doi.org/10.1016/j.marpolbul.2019.110877

Elías, R. (2015). Mar del plástico: una revisión del plástico en el mar. Marine and Fishery Sciences, 27, 83-105.

Erni-Cassola, G., Gibson, M. I., Thompson, R. C., \& Christie-Oleza, J. A. (2017). Lost, but found with Nile red: a novel method for detecting and quantifying small microplastics ( $1 \mathrm{~mm}$ to $20 \mu \mathrm{m}$ ) in environmental samples. Environmental Science \& Technology, 51(23), 13641-13648. https://doi.org/10.1021/acs. est. $7 \mathrm{~b} 04512$

Farrell, P., \& Nelson, K. (2013). Trophic level transfer of microplastic: Mytilus edulis (L.) to Carcinus maenas (L.). Environmental Pollution, 177, 1-3. https://doi. org/10.1016/j.envpol.2013.01.046
Fernández, C. (2014). Variación estacional e interanual de la dieta de la lisa, Mugil cephalus Linnaeus, 1758, frente a Callao $\left(12^{\circ} \mathrm{S}\right)$, en 2005 y 2013. Ecología Aplicada, 13(2), 211-223.

Filgueira, R., Fernández-Reiriz, M. J., \& Labarta, U. (2009). Clearance rate of the mussel Mytilus galloprovincialis. II. Response to uncorrelated seston variables (quantity, quality, and chlorophyll content). Ciencias Marinas, 36(1), 15-28.

Freitas, R., Silvestro, S., Coppola, F., Meucci, V., Battaglia, F., Intorre, L., \& Faggio, C. (2019). Biochemical and physiological responses induced in Mytilus galloprovincialis after a chronic exposure to salicylic acid. Aquatic Toxicology, 214, 105258. https://doi. org/10.1016/j.aquatox.2019.105258

Frias, J. P. G. L., \& Nash, R. (2019). Microplastics: finding a consensus on the definition. Marine Pollution Bulletin, 138, 145-147. https://doi.org/10.1016/j. marpolbul.2018.11.022

GESAMP. (2015). Sources, fate and effects of microplastics in the marine environment: a global assessment. International Maritime Organization.

Goldstein, M. C., Titmus, A. J., \& Ford, M. (2013). Scales of spatial heterogeneity of plastic marine debris in the northeast Pacific Ocean. PloS one, 8(11), e80020. https://doi.org/10.1371/journal.pone.0080020

González, Z. C. A., Vallarino, A., Pérez, J. J. C., \& Low, P. A. M. (2014). Bioindicadores: Guardianes de nuestro futuro ambiental. Editorial El Colegio de la Frontera Sur, México.

Green, D. S. (2016). Effects of microplastics on European flat oysters, Ostrea edulis and their associated benthic communities. Environmental Pollution, 216, 95-103. https://doi.org/10.1016/j.envpol.2016.05.043

Gutiérrez-Díaz, C. M. A. (2017). Alimento y hábitos alimentarios de Sciaena deliciosa "lorna" (Tschudi, 1846) desembarcada en la Región La Libertad durante el año 2016. (Tesis de Grado). Universidad Nacional de Trujillo, Perú. https://hdl. handle.net/20.500.12958/3164

Horton, A. A., Walton, A., Spurgeon, D. J., Lahive, E., \& Svendsen, C. (2017). Microplastics in freshwater and terrestrial environments: evaluating the current understanding to identify the knowledge gaps and future research priorities. Science of the Total Environment, 586, 127-141.

Instituto del Mar del Perú. (2017). Informe Técnico Extraordinario $N^{\circ} 001-2017 / E N F E N$ EL NIÑO COSTERO 2017. Instituto del Mar del Perú.

Inoue, T., \& Yamamuro, M. (2000). Respiration and ingestion rates of the filter-feeding bivalve Musculista senhousia: implications for water-quality control. 
Journal of Marine Systems, 26(2), 183-192. https:// doi.org/10.1016/s0924-7963(00)00053-1

IPCC. (2014). Cambio climático 2014: Informe de síntesis. https://www.ipcc.ch/site/assets/uploads/2018/02/ SYR_AR5_FINAL_full_es.pdf

IPCC. (2018). Summary for Policymakers. https://www. ipcc.ch/sr15

Jørgensen, C. B. (1996). Bivalve filter feeding revisited. Marine Ecology Progress Series, 142, 287-302. https://doi.org/10.3354/meps142287

Kratina, P., Watts, T. J., Green, D. S., Kordas, R. L., \& O'Gorman, E. J. (2019). Interactive effects of warming and microplastics on metabolism but not feeding rates of a key freshwater detritivore. Environmental Pollution, 255, 113259. https://doi. org/10.1016/j.envpol.2019.113259

Lenz, M. (2016). Erhöht Wärmestress die Empfindlichkeit benthischer Filtrierer gegenüber Mikroplastik? Ein Projekt im Rahmen des internationalen Forschungsund Ausbildungsprogramms GAME (Globaler Ansatz durch Modulare Experimente). https://www.geomar. $\mathrm{de} /$ fileadmin/content/forschen/fb3/eoe-b/game/Abschlussbericht_GAME XIV.pdf

Lenz, M., Ahmed, Y., Canning-Clode, J., Díaz, E., Eichhorn, S., Fabritzek, A. G., \& Machura, S. (2018). Heat challenges can enhance population tolerance to thermal stress in mussels: a potential mechanism by which ship transport can increase species invasiveness. Biological Invasions, 20(11), 3107-3122. https://doi.org/10.1007/s10530-018-1762-8

Li, Y., Lu, Z., Zheng, H., Wang, J., \& Chen, C. (2020). Microplastics in surface water and sediments of Chongming Island in the Yangtze Estuary, China. Environmental Sciences Europe, 32(1), 1-12. https:// doi.org/10.1186/s12302-020-0297-7

Li, J., Lusher, A. L., Rotchell, J. M., Deudero, S., Turra, A., Bråte, I. L. N., \& Shi, H. (2019). Using mussel as a global bioindicator of coastal microplastic pollution. Environmental Pollution, 244, 522-533. https://doi. org/10.1016/j.envpol.2018.10.032

Manchala, M., \& Ramana, B. V. (2020). Beat the Plastic Pollution. International Journal of Environmental Planning and Development, 6(2), 1-6. https://doi. org/10.37628/jepd.v6i2.658

Marroni, P. M. S. (2019). Ecología de Diplodon parallelopipedon en Laguna del Sauce y sus implicancias en técnicas de biomanipulación (Tesis de Doctorado). Universidad de la República, Uruguay. https://hdl. handle.net/20.500.12008/24737

Molinet, C., Fuentealba Klenner, M., Arévalo, A., Barahona, N., Asencio, C., Ariz, L., González, J., Cortez, C., Matamala Farran, M., Henríquez Moreira, J., Dorador Parra, C., Valenzuela Oñate, A., Fernández
Cartes, V., Almanza Marroquín, V., Muñoz Klenner, J., Gutiérrez Gallardo, J. C., Díaz Gómez, P., \& Paredes, C. (2007). Diagnóstico biológico-pesquero para recursos bentónicos de la Zona Contigua, $X$ y XI Región. Informe Final. Proyecto FIP $N^{\circ}$ 2005-51. Universidad Austral de Chile, Chile.

Munno, K., Helm, P. A., Jackson, D. A., Rochman, C., \& Sims, A. (2017). Impacts of temperature and selected chemical digestion methods on microplastic particles: Impacts of temperature and digestion method on microplastics. Environmental Toxicology and Chemistry, 37(1), 91-98. https://doi.org/10.1002/etc.3935

Paredes, C., Cardoso, F., Santamaría, J., Esplana, J., \& Llaja, L. (2016). Lista anotada de los bivalvos marinos del Perú. Revista Peruana de Biología, 23(2), 127-150. https://doi.org/10.15381/rpb.v23i2.12397

Paredes, C., Cardoso, F., \& Tarazona, J. (1999). Invertebrados del intermareal rocoso del departamento de Lima, Perú: Una lista comentada de especies. Revista Peruana de Biología, 6(2), 143-151. https://doi. org/10.15381/rpb.v6i2.8309

Pedersen, A. F., Gopalakrishnan, K., Boegehold, A. G., Peraino, N. J., Westrick, J. A., \& Kashian, D. R. (2020). Microplastic ingestion by quagga mussels, Dreissena bugensis, and its effects on physiological processes. Environmental Pollution, 260, 113964. https://doi.org/10.1016/j.envpol.2020.113964

Pérez-Carrasco, L. J. (2017). Alimento y hábitos alimentarios de Paralonchurus peruanus "Suco" (Steindachner, 1875) en la Región de la Libertad durante el año 2016 (Tesis de Grado). Universidad Nacional de Trujillo, Perú. https://hdl.handle.net/20.500.12958/3165

Pinochet, J., Domínguez, J., Neira, E., Rojas, C., Acuña, E., Cancino, J. M., \& Brante, A. (2018). Tidal height and sand as potential drivers of the ecological interaction of the two intertidal mussels Perumytilus purpuratus and Semimytilus algosus. Marine Ecology, 39(4), e12503. https://doi.org/10.1111/maec.12503

Rist, S. E., Assidqi, K., Zamani, N. P., Appel, D., Perschke, M., Huhn, M., \& Lenz, M. (2016). Suspended microsized PVC particles impair the performance and decrease survival in the Asian green mussel Perna viridis. Marine Pollution Bulletin, 111, 213-220. https://doi.org/10.1016/j.marpolbul.2016.07.006

Rist, S., Baun, A., Almeda, R., \& Hartmann, N. B. (2019). Ingestion and effects of micro- and nanoplastics in blue mussel (Mytilus edulis) larvae. Marine Pollution Bulletin, 140, 423-430. https://doi.org/10.1016/j. marpolbul.2019.01.069

Rosa, M., Ward, J. E., \& Shumway, S. E. (2018). Selective capture and ingestion of particles by suspension-feeding bivalve molluscs: a review. Journal of Shellfish Research, 37(4), 727-746. https://doi. org/10.2983/035.037.0405 
Serra, T., Barcelona, A., Pous, N., Salvadó, V., \& Colomer, J. (2020). Synergistic effects of water temperature, microplastics and ammonium as second and third order stressors on Daphnia magna. Environmental Pollution, 115439. https://doi.org/10.1016/j. envpol.2020.115439

Sussarellu, R., Suquet, M., Thomas, Y., Lambert, C., Fabioux, C., Pernet, M. E. J., Le Goïc, N., Quillien, V., Mingant, C., Epelboin, Y., Corporeau, C., Guyomarch, J., Robbens, J., Paul-Pont, I., Soudant, P., \& Huvet, A. (2016). Oyster reproduction is affected by exposure to polystyrene microplastics. Proceedings of the National Academy of Sciences, 113(9), 2430 2435. https://doi.org/10.1073/pnas.1519019113

Sylvester, F., Dorado, J., Boltovskoy, D., Juárez, Á., \& Cataldo, D. (2005). Filtration rates of the invasive pest bivalve Limnoperna fortunei as a function of Size and Temperature. Hydrobiologia, 534(1-3), 71-80. https://doi.org/10.1007/s10750-004-1322-3

Tejada-Pérez, C. A., Villasante, F., Luque-Fernández, C., \& Tejada-Begazo, C. L. (2018). Riqueza y distribución vertical de los moluscos del litoral rocoso de la provincia de Islay, Arequipa, al sur del Perú. Revista Ciencias Marinas y Costeras, 10(1), 47-66. https:// doi.org/10.15359/revmar.10-1.4
Walkinshaw, C., Lindeque, P. K., Thompson, R., Tolhurst, T., \& Cole, M. (2020). Microplastics and seafood: lower trophic organisms at highest risk of contamination. Ecotoxicology and Environmental Safety, 190, 110066. https://doi.org/10.1016/j. ecoenv.2019.110066

Weber, A., Jeckel, N., \& Wagner, M. (2020). Combined effects of polystyrene microplastics and thermal stress on the freshwater mussel Dreissena polymorpha. Science of the Total Environment, 718, 137253. https://doi.org/10.1016/j.scitotenv.2020.137253

Widdows, J. (1978). Combined Effects of Body Size, Food Concentration and Season on the Physiology of Mytilus Edulis. Journal of the Marine Biological Association of the United Kingdom, 58(1), 109-124.

Woods, M. N., Stack, M. E., Fields, D. M., Shaw, S. D., \& Matrai, P. A. (2018). Microplastic fiber uptake, ingestion, and egestion rates in the blue mussel (Mytilus edulis). Marine Pollution Bulletin, 137, 638-645. https://doi.org/10.1016/j.marpolbul.2018.10.061

World Meteorological Organization. (2019). The Global Climate in 2015-2019. Switzerland. 